\title{
Die Krise der Bistumspresse 1989. Bestandsaufnahme und Zukunftsüberlegungen
}

\author{
von Ferdinand Oertel
}

\begin{abstract}
Die Publizistische Kommission der Deutschen Bischofskonferenz hat 1988 das Thema der Bistumspresse neu aufgegriffen, in Arbeits- und Expertengruppen untersuchen lassen und für den 10. und 11. Oktober 1989 in München ein Kolloquium angesetzt, zu dem unter anderem alle Ortsbischöfe eingeladen worden sind. Dabei soll es um eine Ortsbestimmung und um Zukunftsperspektiven der Katholischen Presse, insbesonders der Bistumspresse gehen. „Communicatio Socialis" hat Dr. Ferdinand Oertel um einen Beitrag zum gleichen Thema aus seiner Sicht gebeten. Oertel, der vor genau 40 Jahren, 1949, als Volontär bei der Kölner Kirchenzeitung seinen journalistischen Weg begann, war von 1971 bis 1980 Vorsitzender der Arbeitsgemeinschaft Katholische Presse, Berater der Sach-Kommission VI der Gemeinsamen Synode der Bistümer der Bundesrepublik, bis 1981 Berater der Publizistischen Kommission der Deutschen Bischofskonferenz und hat 1972 in seinem Buch „Dialogforum Kirchenpresse" (Lahn-Verlag, Limburg) die damaligen Überlegungen zur Neugestaltung der Kirchenpresse zusammengefaßt.
\end{abstract}

Die Bischöfliche Studienförderung des Cusanuswerkes veranstaltete im Herbst 1988 eine Tagung über das Thema „Die Kirche und die Medien“. Dabei vertraten die Teilnehmer, etwa 40 junge Doktorandinnen und Doktoranden, gemeinhin ein Reservoir katholischer Elite, einhellig die Auffassung, daß die gegenwärtige Bistumspresse keine Zukunft mehr besitzt. Die jungen Katholiken ordneten die Kirchenzeitungen als Presse einer Institution ein und setzten sie mit der Partei- und Gewerkschaftspresse gleich, die ebenfalls bedeutungslos geworden sei. Gefragt seien katholische Spezialblätter für die verschiedenen Gruppierungen in der Kirche, also zielgruppenorientierte Fachzeitschriften (in der vorwiegend theoretisch geführten Diskussion blieb allerdings die wirtschaftliche Seite des Pressewesens unberücksichtigt).

Die seit 1963 andauernde, jetzt über 25 Jahre währende negative Entwicklung der katholischen Presse in der Bundesrepublik scheint solchen Auffassungen junger Katholiken Recht zu geben. Schon 1975 wurde in dem von der Sachkommission VI der Gemeinsamen Synode der Bistümer in der Bundesrepublik verabschiedeten Arbeitspapier "Kirche und gesellschaftliche Kommunikation“ festgestellt, daß „ die meisten“ kirchlichen und kirchennahen Zeitschriften "sich heute in einer für Fachleute deutlich erkennbaren Krise“ befinden - mit der bemerkenswerten Ergänzung: „die aber weder von der Mehrzahl ihrer Leser noch von allen zuständigen Stellen der Kirche in ihrer ganzen Tragweite wahrgenommen wird".'

Eine nüchterne Zahlenbilanz belegt die Krisensituation, die sich bis heute weiter zugespitzt hat. Als die Bundesrepublik 1949 gegründet wurde, erschienen die 22 Bistumszeitungen mit einer Gesamtauflage von rund 2 Millionen Exemplaren. 15 Jahre später, Ende 1963 - während der Zeit des Zweiten Vatikanischen Konzils - erreichte die Bistumspresse mit 2,4 Millionen Auflagen ihren Höchststand. Zehn Jahre später, 1974 zur Zeit der Gemeinsamen Synode - hatten die 22 Bistumszeitungen schon einen Auflagenrückgang von einer halben Million, also 20 Prozent, zu beklagen und verzeichneten noch knapp 1,9 Millionen Gesamtautlage. Weitere 15 Jahre später, Ende 1989 und insgesamt 25 Jahre nach dem Höchststand während des Konzils, ist die Gesamtauflage auf 1,5 Millionen gesunken.

Die Auflagenentwicklung der einzelnen Bistumsblätter ist zwar unterschiedlich. So verzeichnen die Bistumsblätter von Limburg und Essen seit ihren Höchstauflagen zur 
Konzilszeit bis 1989 über 60 Prozent Rückgang, während die Blätter von Eichstätt mit 20 und Münster mit 17 Prozent den geringsten Verlust aufweisen. Verbunden mit dem Auflagenrückgang ist ein pastoral stark ins Gewicht fallender Rückgang der Reichweite, und dieser ist nicht einfach prozentual auf die katholische Gesamtbevölkerung verteilt, sondern betrifft vor allem die jüngeren und mittleren Generationen. So hat eine 1987 von Media Markt Analysen, Frankfurt, im Auftrag von 15 Bistumsblatt-Verlagen durchgeführte Funktionsanalyse ergeben, daß über die Hälfte ( $52 \%$ ) aller Abonnenten über 60 Jahre und drei Viertel schon über 50 Jahre alt sind. ${ }^{2}$ Dabei ist der hohe Auflagenrückgang nicht nur, wie bereits das Arbeitspapier der Synode anmerkt, „parallel zur Kurve des Rückgangs der Kirchenbesucher“ verlaufen, sondern auch trotz zahlreicher Verbesserungsmaßnahmen, die in den Jahren nach dem Konzil von Bistumszeitungen gemeinsam oder einzeln erfolgt sind.

Bei einer Bestandsaufnahme und Situationsanalyse der Bistumspresse stellen sich Fragen in zweifacher Richtung: Erstens rückblickend, was von den Anfang der 70er Jahre gefaßten Maßnahmen verwirklicht worden ist, was nicht und warum nicht; und zweitens auf die Zukunft hin, ob neue Maßnahmen möglich sind, wie sie aussehen müßten und ob sie zu verwirklichen sind. Damit diese Fragen beantwortet werden können, müssen einige Eckdaten der Vergangenheit ins Gedächtnis zurückgerufen werden.

\section{Funktionswandel nach Konzil und Synode}

Nachdem die Kirche bis in die Mitte dieses Jahrhunderts ein vielfältig gespaltenes, durchwegs eher negatives Verhältnis zu den Medien gehabt hat (indem sie zunächst fast ausschließlich die Gefahren der Presse und später auch des Films in den Blick rückte), wurden für ein neues Verhältnis zu den Medien das Zweite Vatikanische Konzil und insbesondere die 1971 erschienene Pastoralinstruktion "Communio et progressio“ (CeP) wegweisend. Das Konzil hatte erstmals für den Bereich der weltlichen Ordnung eine eigene Sachgerechtigkeit festgestellt und die Sachkompetenz für den „Aufbau der zeitlichen Ordnung “ den Laien zuerkannt. Damit hatte das Konzil auch einen Wandel der Funktion der Kirchenpresse in Gang gesetzt, der Ende der 60er und Anfang der 70er Jahre intensiv unter den Herausgebern, Verlegern und Redakteuren der Kirchenpresse diskutiert und von Publizistikwissenschaftlern begleitet worden ist. ${ }^{3}$

Verkürzt und schlagwortartig zusammengefaßt, galt Kirchenpresse bis dahin als „Sprachrohr des Bischofs“ oder „Zweite Kanzel“, also als reines Instrument der kirchlichen Verkündigung. Die Redaktionen aller Bistumszeitungen wurden bis Anfang der 60 er Jahre traditionsgemäß von Priestern geleitet. Neben der als amtlich verstandenen Verbreitung der kirchlichen Lehre und einem zur sittlichen Erbauung und moralischen Festigung dienenden Unterhaltungsteil hatten die Bistumszeitungen seit ihrer Wiederbegründung nach dem Zweiten Weltkrieg auch die Nachrichtenübermittlung über das kirchliche Leben übernommen, weil keine katholischen Tageszeitungen mehr wiedererstanden waren und dieser Bereich von der anderen Tagespresse nur ungenügend behandelt wurde.

Aus der Anerkennung der Eigengesetzlichkeit der weltlichen Dinge folgerte für die kirchliche Presse, daß auch sie zunächst ihrem Wesen nach Presse ist, demnach den Gesetzmäßigkeiten der Presse unterliegt und nach ihnen gestaltet werden muß. Ihre Teilnahme am Verkündigungsauftrag muß in pressespezifischer Weise erfolgen.

Die Programmatik des Funktionswandels der Bistumspresse ist in mehreren Dokumenten niedergelegt, die Anfang der 70er Jahre erschienen. Die „Arbeitsgemeinschaft Katholische Presse“ (AKP) verabschiedete auf ihrer Mitgliederversammlung in Mainz 
am 27. Januar 1972 „Grundsätze für einen Rahmenplan der Kirchenpresse“. ${ }^{4}$ Die gemeinsame Synode der Bistümer in der Bundesrepublik diskutierte auf der MaiVollversammlung 1972 ein Papier „Grundsätze für ein Gesamtkonzept der kirchlichen Publizistik“. ${ }^{5}$ Die Deutsche Bischofskonferenz gab im Februar 1972 ein Gutachten "Katholische Publizistik“ in Auftrag, das unter der Leitung von Prof. Dr. Michael Schmolke erstellt wurde und dessen Ergebnisse in einem zusammenfassenden Bericht vom März 1974 vorliegen. ${ }^{6}$ Die Sachkommission VI der Gemeinsamen Synode verabschiedete am 20. Juni 1975 das bereits erwähnte Arbeitspapier „Kirche und gesellschaftliche Kommunikation“.

In den „Grundsätzen für einen Rahmenplan der Kirchenpresse" waren u.a. Richtlinien für die Bistumspresse enthalten, die bereits im Oktober 1969 dem damaligen Referenten für Pressefragen der Publizistischen Kommission, Bischof Heinrich Tenhumberg, zur Genehmigung „ad experimentum“ durch die Deutsche Bischofskonferenz übermittelt worden waren; ferner Anregungen für eine Feldbefragung über die Kommunikations-und Informationserwartungen an die Kirchenpresse sowie ein Arbeitspapier zur Einrichtung einer Gemeinschaftsredaktion für die Kirchenpresse (Bistumspresse). Die neue Funktion der Bistumspresse wird folgendermaßen umschrieben:

„Die Kirchenzeitung (Bistumsblatt) versteht sich als eine Zeitung der Diözesankirche, die alle Gläubigen - Bischöfe, Priester, Laien - miteinander verbindet. Sie muß den Informationsfluß zwischen allen Ämtern und Gruppen der Kirche untereinander ermöglichen und so den Kommunikationsprozeß in der Ortskirche pressegerecht fördern. Daraus ergibt sich der Anspruch auf umfassende Information und der Auftrag, Forum einer ungehinderten und kritischen Meinungsbildung innerhalb der Kirche zu sein“".

In seinem Gutachten „Katholische Publizistik“ stellt Michael Schmolke (zitiert nach dem zusammenfassenden Bericht von März 1974) zunächst fest:

„Kirche und Katholiken in der Bundesrepublik Deutschland brauchen

a) eine spezifische ,Massenpresse;, d.h. mindestens starke Bistumszeitungen

b) Organe für die Führungsschicht, und zwar möglichst nicht nur eines ...

c) mindestens eine Unterhaltungszeitschrift (genaue Zielgruppe aber noch nicht bekannt (Junge Familie?)".

Über die Bistumszeitungen heißt es weiter in dem Gutachten: „Sie sind immer noch das wichtigste und geeigneteste Organ diözesaner Kommunikation." Infolge der schon damals im Gutachten diagnostizierten „essentiellen Krise“ der Bistumspresse wird vor allem eine „Feldbefragung“ über die Erwartung an die Kirchenpresse vorgeschlagen sowie eine „forcierte Zusammenarbeit der einzelnen Redaktionen“.

In der Frage der Kooperationen greift Schmolke den Vorschlag der AKP zur Bildung einer Gemeinschaftsredaktion auf und modifiziert ihn. In einer „kleinen Lösung“ schlägt er der Bischofskonferenz vor, zunächst zwei regionale Gemeinschaftsredaktionen mit Hilfe überdiözesaner Mittel zu gründen und diese Redaktionen als Modellversuche für drei Jahre finanziell zu fördern. Als „große Lösung“ werden vier Regionalredaktionen vorgeschlagen.

Die Idee der regionalen Gemeinschaftsredaktionen wird von Schmolke damit begründet, daß es kaum realisierbar erscheine, die einzelnen Redaktionen so auszustatten, daß sie alle Aufgaben wahrnehmen können. Aus demselben Grund war auch von der AKP die Einrichtung einer Gemeinschaftsredaktion vorgeschlagen worden. In einem Arbeitspapier vom 13. April 1972 heißt es, daß weder gelegentliche Kooperationen von Redaktionen noch die Katholische Nachrichten Agentur KNA alleine die spezifischen Funktionen übernehmen können, die für die neue Konzeption der Kirchenpres- 
se (Bistumspresse) notwendig sei. Die Gemeinschaftsredaktion sollte gezielt die überdiözesanen Themen, angefangen von eigenen Nachrichtenerfassungen über Kommentare, eigene Artikelserien und Reportagen bis hin zum Feuilleton erstellen. Schon damals wird betont, daß dadurch die einzelnen Redaktionen der Bistumszeitungen entlastet werden, um die diözesane Berichterstattung verstärkt wahrzunehmen. ${ }^{10}$

Damit die Kirchenpresse (Bistumspresse) ihre neue Aufgabe und Funktion hinreichend wahrnehmen kann, wird in allen Papieren - denen der AKP, denen der Synode und in den Gutachten - eine Reihe grundsätzlicher Bedingungen und Maßnahmen genannt:

a) Redaktionen und Verlage müssen personell und materiell so ausgestattet sein, daß sie ihren Auftrag pressespezifisch erfüllen können;

b) besonderer Wert ist deshalb auf die Nachwuchsförderung qualifizierter katholischer Journalisten zu legen;

c) die Erwartungen an die Kirchenpresse sind durch eine Umfrage zu erkunden;

d) die Katholische Nachrichten Agentur KNA muß weiter ausgebaut werden (unabhängig von einer Gemeinschaftsredaktion, die andere Aufgaben als eine Agentur habe);

e) zur Intensivierung sämtlicher Fördermaßnahmen wird die Einrichtung einer Kontaktstelle der Bischofskonferenz für Verlage und Redaktionen vorgeschlagen.

In allen Dokumenten spielt die Frage der Subventionen durch die Bischöfe eine entscheidende Rolle. In den „Grundsätzen für einen Rahmenplan“ der AKP heißt es: „Da die Kirchenpresse ihrer Aufgabenstellung nach am Wesensauftrag der Kirche, der Heilsverkündigung, teilnimmt, können kirchliche Presseorgane jedoch nicht nur unter wirtschaftlichen Gesichtspunkten betrachtet und geführt werden. Ausschlaggebend ist ihre kirchliche Effizienz". Zunächst wird betont, daß die in der AKP zusammengeschlossenen Organe es „für ihre Pflicht (halten), durch sorgfältige und fachgerechte verlegerische Planung und Kalkulation die für ihre Aufgabenerfüllung notwendigen Mittel soweit wie möglich selbst zu erbringen". Aber dann folgt die Feststellung: "Wenn erforderlich, müssen jedoch kirchliche Zuschüsse bereitgestellt werden. " lerdings sollten Zuschüsse nicht der Finanzierung einzelner Organe dienen, sondern zur Verbesserung der Grundlagen für die gesamte Kirchenpresse.

Michael Schmolke betont in seinem Gutachten, daß „die Kirche sich m.E. mit Anregungen und finanziellen Mitteln im Bereich der Publizistik nur dort engagieren (kann), wo der Wille der Dienstleistung im Sinne der gesellschaftlichen Funktionen der Kirche hinreichend dokumentiert wird“. Dazu zählt er die Bistumsblätter. Stützungsmaßnahmen sollten dabei aber ${ }_{\text {}}$ allen Redaktionen... durch die Strukturreformen zugute kommen ${ }^{*}{ }^{12}$

Im Arbeitspapier der Synoden-Sachkommission VI wird gefordert, daß die Kirchenpresse in ihrer Krisensituation „vom Verantwortungsbewußtsein der ganzen Kirche mitgetragen werden (sollte). Das bedeutet, je nach den Umständen, auch finanzielle Förderung. ${ }^{13}$

Zum Einsatz finanzieller Subventionen hatten die Bischöfe sich 1972 auch ausdrück. lich verpflichtet, als nach der Einstellung der Finanzmittel für die neue katholische Wo. chenzeitung „Publik“ in der Gemeinsamen Synode vehement ein neues Engagement für die katholische Presse gefordert worden war. Am 11. Mai 1972 verkündete Weihbischof Georg Moser auf der Gemeinsamen Synode ein "Sofortprogramm“, das alle damaligen Forderungen auf dem Mediensektor aufgriff: von der Verbesserung der Kir- 
chenpresse und der KNA über die Einrichtung einer Kontaktstelle für die Presse, die Zusammenführung aller publizistischen Hauptstellen und die Förderung des journalistischen Nachwuchses bis zur Errichtung eines Dokumentationszentrums. ${ }^{14}$

\section{Bilanz nach 20 Jahren}

Eine Bilanz dieser Planungen, Vorstellungen und Forderungen kann wiederum nur kurz und schlaglichtartig ausfallen und sich nur auf die Dinge beziehen, die die Bistumspresse betreffen oder berühren.

1.) 1974 wurde von der Bischofskonferenz eine „Kontaktstelle Verlagskooperation“ gegründet, aus der ein Jahr später die Medien-Dienstleistungs-Gesellschaft MDG entstand. In der Satzung der MDG werden deren Aufgaben beschrieben: „Bestandsaufnahmen, Analysen, Entscheidungshilfen sowie die Entwicklung von Kooperationsprojekten, die Zurverfügungstellung von personellen und sachlichen Diensten auf verlegerischen, redaktionellen, wirtschaftlichen und technischen Sektoren des Medienbereiches. ${ }^{\text {"15 }}$ Hingegen soll die MDG selbst nicht unternehmerisch tätig werden.

In enger Zusammenarbeit mit den Gremien der AKP hat die MDG von Anfang an ein Hauptaugenmerk auf die vorgeschlagenen und neue Verbesserungsmaßnahmen für die Kirchenpresse gerichtet. Diese Maßnahmen umfassen redaktionelle Inhaltsanalysen und Betriebsvergleiche, Fortbildungskurse für Redakteure und Management-Training, Werbemittel und Vertriebssysteme, Kooperationsmaßnahmen und Objektentwicklungen. Dabei hatte (und hat) die MDG einerseits auch gegen Vorurteile und Verkrustungen im etablierten kirchlichen Pressewesen zu kämpfen, andererseits mußte sie die Erfahrung machen, daß Methoden des säkularen Pressemarktes nicht ohne weiteres auf den kirchlichen Pressebereich erfolgreich übertragen werden können.

2.) 1974 begannen auch die Vorarbeiten für eine groß angelegte Feldbefragung, die von Januar bis Mai 1975 vom Institut für Kommunikationsforschung (IFK) in Wuppertal durchgeführt wurde. ${ }^{16}$ Sie umfaßte eine Repräsentativbefragung über das Informationsverhalten der Katholiken und ihre Erwartungen gegenüber Kirche und Medien generell und der Kirchenpresse im besonderen, ferner eine titelbezogene Umfrage in den Diözesen bei Beziehern der Kirchenzeitung und auch bei Abbestellern (Nichtlesern). Die beiden wichtigsten Ergebnisse faßte Michael Schmolke im Kommentar zur Feldbefragung folgendermaßen zusammen:

a) „Kirchenpresse, speziell Bistumspresse, ist vorwiegend kircheninterne Kommunikation. Sie erreicht hauptsächlich die Kirchgänger.

b) Die Leserschaft ist tatsächlich 'alt'“. ${ }^{17}$

Die AKP zog für ihre Überlegungen und Planungen folgende weitere Schlüsse aus den Ergebnissen:

a) „Die Erwartungen an die Bistumspresse ... beziehen sich neben einem Vorrang für kirchliche Themen vor allem auf Lebenshilfe besonders für alte Leute, Ehe und Familie, während politische Beiträge noch hinter die Unterhaltung zurückfallen.

b) Leser und Nichtleser treffen sich in der Feststellung, daß die Kirchenpresse 'weltoffener' berichten müsse“ ${ }^{4}{ }^{18}$

Eine systematische Umsetzung der Ergebnisse ist nicht erfolgt. Zwar sind in einzelnen Redaktionen inhaltliche Konsequenzen gezogen worden, aber es wurde weder eine neue Gesamtkonzeption entwickelt noch im Hinblick auf regional unterschiedliche $\mathrm{Si}$ tuationen eine modifizierte Redaktionskooperation verwirklicht. Unter Einbeziehung 
von Ergebnissen der Feldbefragung legten AKP und MDG 1977 wohl ein „Projekt Modell-Entwicklung Kirchenpresse ${ }^{* 19}$ vor, auf das im nächsten Punkt näher eingegangen wird.

3.) Die Gemeinschaftsredaktion ist weder in der von der AKP geplanten Form noch in den beiden von Michael Schmolke den Bischöfen vorgeschlagenen Formen einer kleinen oder großen Lösung verwirklicht, nicht einmal ernsthaft angegangen worden. Abgesehen von wirtschaftlichen Fragen, richteten (und richten) sich die Hauptbedenken von Verlegern und Redakteuren gegen Gemeinschaftsredaktionen, weil einerseits im Hintergrund das Gespenst einer Einheitskirchenzeitung befürchtet wird (wodurch die Bistumsblattregionen und -verlage sich auf regionale Funktionen reduziert fühlen könnten), andererseits selbst für vier (theoretisch geplante) regionale Gemeinschaftsredaktionen in der diözesanen und überdiözesanen Realität keine Basis gegeben ist. Nicht nur das „Erbhofdenken “, das Schmolke schon 1974 in seinem Gutachten „Katholische Presse " erwähnt, ${ }^{20}$ behindert überdiözesane Lösungen, sondern auch schwer oder praktisch gar nicht zu ändernde Besitzverhältnisse, Herausgeber- und Verlagskonstruktionen und andere traditionelle Gegebenheiten und Gewohnheiten im Gesamtbereich der Bistumspresse stehen ihnen entgegen, von Fragen der Konzeption und der persönlichen Leistungsfähigkeit (und Einschätzung) ganz abgesehen. Das von der AKP gemeinsam mit der MDG erarbeitete „Projekt Modellentwicklung Kirchenzeitung “ versuchte, einige dieser Gegebenheiten zu berücksichtigen. Angesichts der Verschiedenartigkeit der Produkte der 22 Bistumszeitungen, die zum Teil durch Bindungen an Druckereien vorgegeben ist, werden drei Modelle vorgeschlagen:

a) im Stil einer Wochenillustrierten

b) im Stil einer Wochenschrift im Buchdruckverfahren

c) im Stil einer Wochenzeitung im Buchdruckverfahren im großen Zeitungsformat. ${ }^{21}$

Bevor auf die tatsächliche Kooperationsentwicklung eingegangen wird, muß auch bei diesem Projekt lapidar festgestellt werden, daß diese drei Modelle im einzelnen nie weiterverfolgt worden sind.

Die MDG hat dagegen schon sehr früh die Idee eines Supplements entwickelt. In einer solchen Beilage im Farbdruck (zur Image-Aufbesserung) und mit Farbanzeigen (zur Mitfinanzierung) sollen überdiözesane Themen integriert werden, was den Einzelredaktionen wiederum mehr Raum für ihre Bistumsberichterstattung einbrächte. Dazu wiederum die lapidare Feststellung: Auch ein solches Supplement ist bisher über das Stadium von Null-Nummern nicht hinausgekommen (mit Ausnahme des 1985 aus Eigeninitiative erwachsenen "Familienjournals" - vgl. Punkt 6f., S. 37).

4.) Die personelle Situation in den Redaktionen der Bistumszeitungen hat sich nur unwesentlich verbessert, obwohl die Bemühungen um die Nachwuchsförderung zu den erfolgreichsten Entwicklungen im kirchlichen Medienbereich der letzten 20 Jahre zählen. Das 1969 gegründete Institut zur Förderung des publizistischen Nachwuchses kann mit beachtenswerten Zahlen über ausgebildete katholische Journalisten aufwarten, doch von diesen sind im Vergleich zur Gesamtzahl nur wenige in die Kirchenpresse gegangen. Es war Zwar von Anfang an nicht das Ziel der katholischen Journalistenausbildung, Nachwuchs für die eigene Presse heranzubilden, aber ein solcher Nachwuchs sollte auch nicht grundsätzlich ausgeschlossen sein, zumal die Verlage der Bistumszeitungen die Mehrzahl der Volontärstellen zur Verfügung stellen (was bei der sowieso knappen Redaktionsbesetzung keineswegs immer ideale Voraussetzungen für eine Volontärausbildung bietet). Von 1969 bis 1989 verzeichnet das Institut $298 \mathrm{Absol}$ venten, wovon ohnehin nur weniger als die Hälfte ( 144) in der Presse tätig sind, und davon wiederum nur 30 als Vollredakteure in der Kirchenpresse. ${ }^{22}$ 
Wie sieht die personelle Entwicklung tatsächlich aus? Eckhardt Bieger führt in seiner Untersuchung über die „Redakteure der Bistumspresse“ 1975 insgesamt 82 Redakteursplanstellen bei den 22 Bistumsblättern auf. ${ }^{23}$ Nach dem Mitgliedsverzeichnis der $\mathrm{AKP}^{24}$ sind in den Redaktionen der 22 Bistumsblätter zur Zeit 92 Redakteure beschäftigt, also nur zehn mehr. Und obwohl in der grafischen Gestaltung und in der Ausstattung der Bistumszeitungen in den letzten 20 Jahren erhebliche Verbesserungen festzustellen sind, weisen nur drei oder vier der Bistumszeitungen einen eigenen Grafiker auf (in anderen Redaktionen werden gewiß auch grafische Abteilungen von Druckereien hinzugezogen, aber noch längst nicht in allen Redaktionen).

5.) Zur Katholischen Nachrichten Agentur KNA hatte Michael Schmolke in seinem Gutachten schon 1974 festgestellt: „Für die Bistumspresse ist die KNA zur Zeit unentbehrlich, wobei gefragt werden muß, ob die Leistungen der KNA die für die Bistumspresse am besten geeignete Form und Qualität haben. ${ }^{23}$ Otto B. Roegele stellte in einer Bilanz des „Sofortprogramms“ der Deutschen Bischofskonferenz fünf Jahre nach dem Ende der Synode fest, daß der „großzügige Ausbau der Katholischen NachrichtenAgentur noch aussteht, aber nach den Umständen muß man wohl froh sein, daß diese Institution von den Sparmaßnahmen der jüngsten Zeit nicht stärker betroffen wurde. ${ }^{\text {“24 }}$ In den 80 er Jahren ist die KNA so ausgestattet worden, daß sie im technischen Bereich immer mit der Entwicklung Schritt halten konnte und im Bereich der katholischen Presse durchaus als Vorreiter und Schrittmacher gilt. Im inhaltlichen Bereich ist versucht worden, ein besseres und spezielles Angebot für die Kirchenpresse zu entwikkeln; doch bis heute gibt es keinen gesonderten Basisdienst für die Bistumspresse mit eigenen Nachrichtenfassungen. Abgesehen von gelegentlichen Zusammenfassungen einzelner kirchlicher Ereignisse sind die Bistumszeitungen auf die aktuellen Tagesmeldungen der KNA angewiesen, die in gleicher Fassung auch allen Rundfunkanstalten und Tageszeitungen zugehen.

6.) Im Bereich der redaktionellen und verlegerischen Kooperationen sind drei Entwicklungen zu nennen. Schon 1971 hatten die Bistumszeitungen von Limburg und Fulda mit einer Kooperation begonnen, die 1976 auf Mainz ausgeweitet wurde. Seither erscheinen diese drei Bistumszeitungen in einem Verlag, haben eine Zentral- und drei Diözesanredaktionen. Sie produzieren einen gemeinsamen Teil und haben jeweils eigene Bistumsteile.

Einige Jahre später begannen die Bistumszeitungen von Hildesheim und Berlin eine Kooperation, der sich später Osnabrück und schließlich auch Paderborn anschlossen. Auch diese Kooperation besteht in der redaktionellen Erstellung von überdiözesanen Seiten und in ihrer gemeinsamen Produktion. 1986 haben diese vier Bistumszeitungen ein monatliches Farbsupplement mit dem Titel „Familienjournal“ entwickelt, das einerseits als thematische Ergänzung der Trägerblätter und zugleich als Entlastung für die Bistumsberichterstattung sowie andererseits als qualitative Bereicherung mit der Möglichkeit des Vierfarbdruckes und der Aufnahme von Vierfarbanzeigen geplant war. Die Einführung dieser monatlichen Beilage hat Bewegung in den Supplement-Bereich der Bistumspresse gebracht, auf dem es zuvor bereits die Kombination mehrerer anderer Bistumszeitungen mit dem Familienmagazin „Leben \& Erziehen“ gegeben hatte. Als die Überalterung der Leser der Bistumspresse jedoch fortschritt, bildeten die Leser der Bistumszeitungen nicht mehr die Zielgruppe von „Leben \& Erziehen“, so daß Kooperationsgespräche zwischen den Verlagen und Redaktionen des „Familienjournals“ und „Leben \& Erziehen " stattfanden. Sie führten dazu, daß 1988 die Kombination von „Leben \& Erziehen“ mit Bistumszeitungen ganz beendet und stattdessen in Aachen und zusätzlich in Rottenburg-Stuttgart das „Familienjournal“ der vier norddeutschen Blätter übernommen wurde. 
Das „Familienjournal“ war nicht zuletzt auf Grund von Ergebnissen der Funktionsanalyse der 15 Bistumsblattverlage von 1987 konzipiert worden. Die Untersuchung hatte die fortschreitende Überalterung der Leser bestätigt und ein Auseinanderklaffen der Erwartungen an die Bistumspresse aufgewiesen. Während die älteren Leser vorwiegend Klarheit und Bestätigung der kirchlichen Lehre erwarten, wünschen die Angehörigen der mittleren und jüngeren Generation eine argumentative Diskussion von Glaubensproblemen und insbesondere praktische Lebensorientierung und Lebenshilfe. ${ }^{25}$

Bei der Planung des „Familienjournals" war es immer schon als Endziel angesehen worden, eine wöchentliche Beilage mit einem Fernsehprogramm zu erreichen. Die Bedeutung des Fernsehens sowohl als Informationsquelle als auch in der Themensetzung des Alltags war zwar bereits ein wichtiges Ergebnis der Feldbefragung von 1975; in den Konzeptionsdiskussionen der Kirchenpresse geriet es aber in Vergessenheit. Die meisten Bistumszeitungen veröffentlichen seit langem die vom Kölner Institut für Medieninformation altersstufenmäßig gekennzeichneten Programme der beiden öffentlichrechtlichen Anstalten; seit der Ausweitung des Programmangebotes vor allem durch die neuen privaten Anstalten hat sich die Situation aber stark verändert. Aus pastoraler Sicht erscheint eine umfassende Programm-Orientierung mit einer medienpädagogischen Begleitung wünschenswert. Den Bistumszeitungen sind jedoch Grenzen für eine angemessene Ausweitung des Programmteils und einer zusätzlichen medienkritischen Begleitung gesetzt, so daß plötzlich die Frage einer Fernsehbeilage akut geworden ist.

In Abstimmung mit der Zentralstelle Medien im Sekretariat der Deutschen Bischofskonferenz entwickelt die MDG zusammen mit Vertretern der Verleger und Chefredakteure der Bistumspresse zur Zeit eine solche Fernsehbeilage. Sie soll im Programmteil und in Begleitartikeln eine qualifizierte Orientierung aus christlichem Wertverständnis bieten (ohne moralischen Zeigefinger und Bevormundung). Um die Frage zu beantworten, ob ein solches Fernseh-Supplement (abgesehen von der Frage der Finanzierbarkeit) zur Stabilisierung der Bistumspresse beitragen kann, müssen zunächst noch einige weitere Punkte grundsätzlicher Art erörtert werden.

\section{Abschied von den Prinzipien Bistumsorgan und Dialogforum?}

Wenn über die Bistumspresse gesprochen wird, muß differenziert werden. Rein zahlenmäßig betrachtet, haben elf (die Hälfte) der Bistumszeitungen Auflagen, die unter 60.000 liegen, also marktwirtschaftlich kaum noch geführt werden können. Sieben weitere Blätter liegen mit ihren Auflagen zwischen 60.000 und 100.000, und nur vier kommen noch auf über 100.000 (Münster, Köln, Freiburg, Paderborn). Über die Hälfte aller Bistumszeitungen erhalten Subventionen in der einen oder anderen Form. Alle Maßnahmen der letzten zwanzig Jahre haben zu keiner Stabilisierung geführt. Auf Grund dieses Tatbestandes, aber auch auf Grund der veränderten Situation im kirchlichen Leben und im gesamten Medienbereich habe ich auf der Tagung der Bischöflichen Studienförderung des Cusanuswerkes über „Die Kirche und die Medien“ im Herbst 1988 u.a. folgende grundsätzliche Fragen gestellt:

- Ist das Thema Kirche in unserer Gesellschaft noch so gefragt, daß Kirchenzeitungen noch „zu verkaufen" sind - im doppelten Sinne: vom Leserbedürfnis her und wirtschaftlich?

- Genügt den meisten Menschen, die noch am kirchlichen Leben teilnehmen, nicht dasjenige an Informationen über Kirche und Glauben, was in Fernsehen, Hörfunk und säkularen Zeitungen veröffentlicht wird? 
- Stimmt das Konzept der Bistumspresse noch, a ll e n Gläubigen im Bistum a l l e s in gleicher Weise bringen zu müssen?

- Reichen für innerkirchliche Informationen die Pfarrblätter und die Berichterstattung in den Lokalmedien aus?

- Hätten Kirchenzeitungen eine bessere Chance, wenn sie einen freien und offenen Dialog führen könnten?

- Können Lesererwartung und Markt entscheidend sein, wenn kirchliche Presse am Verkündigungsauftrag teilhat?

- Muß die Kirche dafuir ggf. Subventionen geben und nach welchen Kriterien?

- Haben nicht alle Kirchensteuerzahler (=Mitglieder) Anrecht auf regelmäßige kostenlose Informationen aus der Kirche?

- Könnte kirchliche Medienarbeit nur dann wirkungsvoll sein, wenn die Kirche selbst ihre Verkündigung und Pastoral „verbessert“?

Auf diesem breiteren Hintergrund der Kommunikation der Kirche müssen für die Bistumspresse auch zentrale Fragen angegangen werden: ob das Prinzip „Organ der Ortskirche“ und das Prinzip „Dialogforum“ unverändert beibehalten werden können?

Zum Prinzip des „Organs der Ortskirche“: Selbst wenn man es von der Theologie der Ortskirche her für wünschenswert ansehen kann, daß es ein eigenes Bistumsorgan gibt, muß gefragt werden, ob die jetzige Form und Ausprägung die einzig mögliche ist, vor allem dann, wenn sie wirtschaftlich problematisch und in ihrer pastoralen Effizienz stark eingegrenzt worden ist, ganz abgesehen davon, daß heute auch bei vielen Kirchgängern nicht die Bistums-, sondern die Gemeindezugehörigkeit im Vordergrund steht.

Die gegenwärtigen Bistumszeitungen sind ohnehin nicht aus einer Theologie der Ortskirche entstanden, sondern unter dem Z wang der nationalsozialistischen Regierung, die 1936 im kirchlichen Bereich nur noch ein offizielles Verkündigungsorgan im Bistum zuließ und dadurch zur Zusammenlegung vieler regionaler Kirchenzeitungen oder zu Neugründungen führte. Unter den 77 „populären religiösen Zeitschriften und Sonntagsblättern“, die der „Katholische Literaturkalender 1926“ aufführt, finden sich nur acht Diözesanzeitungen. ${ }^{26}$ Im Ausland liegt die Situation der Katholischen Presse gleichfalls anders. Auch in ausgesprochen katholischen Ländern Europas (Ausnahme: Österreich) oder in Ländern der westlichen Welt (ausgenommen USA) mit einer Vielzahl von Bistümern sind Bistumszeitungen in der deutschen Form die Ausnahme. Von einem „Prinzip Organ der Ortskirche“ kann daher nicht gesprochen werden. Wenngleich ein Bistumsorgan zweifellos der Stärkung der Ortskirche dienen kann, verliert es diese Funktion mit sinkender Auflage und wachsender Überalterung. Daraus soll keineswegs die Konsequenz gezogen werden, die existierenden Bistumszeitungen nicht zu erhalten, wohl bedarf ihr Stellenwert der Absolutheit einer Korrektur.

Von daher eröffnen sich nicht nur neue Wege für überdiözesane Kooperationen mit Diözesanteilen, sondern auch für Überlegungen über neue zielgruppenorientierte katholische Zeitschriften. Interessanterweise gehen Überlegungen der evangelischen Kirchengebietspresse in dieselbe Richtung. Friedrich Schwaneke schreibt in seinem Artikel "Weiter bröckeln lassen oder etwas unternehmen?" in den Evangelischen Kommentaren u.a.: „Die Eigenschaft als Landeskirche, als verfaßtes Kirchengebiet also, ist das schwächste Motiv für die Kirchenbildung ... Wenn das schon in Bezug auf die Institution Evangelische Kirche so ist, wie sollte der Kirchengebietsaspekt die Leser-BlattBindung fördern oder gar Abonnenten-Nachwuchs motivieren? Es wird nicht nach Gebieten gefragt und auch nicht nach Institutionen, sondern nach Inhalten. Die großen 
Gesprächsthemen des Protestantismus haben mit Kirchengebieten so gut wie nichts zu tun. ${ }^{27}$

Die evangelischen Kirchengebiete der Landeskirchen sind nicht ohne weiteres mit den katholischen Bistümern zu vergleichen, doch das inhaltliche Interesse an kirchlichen und religiösen Zeitthemen ist auch im katholischen Bereich überdiözesan.

Eine Korrektur in der Konzeption der Bistumspresse erscheint auch in anderer Hinsicht notwendig. Es fragt sich, ob man nicht Abschied nehmen muß vom Prinzip des Dialogforums, zumal in der Form, in der es von der AKP konzipiert worden ist, aber nie verwirklicht werden konnte.

Für das AKP-Konzept sind der Forumscharakter und die dialogische Gestaltung zwar dem Konzil, insbesondere der Pastoralinstruktion „Communio et progressio“ (CeP) entnommen; doch der Kontext der Basis für Forum und Dialog in der Katholischen Kirchenpresse ist weniger in Betracht gezogen worden. CeP stellt fest, daß die neue Technik die Menschen "sozusagen um einen runden Tisch" versammelt, betont das Recht auf Information und Freiheit der Meinungsäußerung auch in der Kirche sowie die Notwendigkeit des ,innerkirchlichen Dialogs“ und des „Dialogs zwischen Kirche und Welt" (wie die entsprechenden Kapitelüberschriften lauten) ${ }^{28}$ Dies alles gilt jedoch unbeschadet vom kirchlichen Auftrag der unverkürzten Verkündigung der Glaubenswahrheit und der Lehre der Kirche. Die entsprechenden Ausführungen in $\mathrm{CeP}$ lauten wörtlich:

„Die katholische Presse öffnet sich der ganzen Welt. Sie bringt Nachrichten, Kommentare und Meinungen über alle Aspekte des heutigen Lebens; sie greift alle Schwierigkeiten und Probleme auf, mit denen der Mensch heute konfrontiert ist; all dies aber im Lichte christlicher Lebensauffassung ... Sie soll zugleich Spiegel sein, der die Welt reflektiert, und Licht, das ihr den Weg zeigt. Sie soll Forum für Begegnung und Gedankenaustausch sein."

Dann heißt es jedoch eindeutig: „Wenn Tagesereignisse Fragen aufwerfen, die das Wesen des christlichen Glaubens berühren, werden katholische Journalisten bemüht sein, sie im Sinne des kirchlichen Lehramtes zu interpretieren ... Katholische Zeitungen, die als offizielle Organe des kirchlichen Lehramtes gelten, obliegt es ..., alle Mühe darauf $\mathrm{zu}$ verwenden, um die Auffassungen jener Stellen und Institutionen darzulegen und deutlich zu machen, deren öffentliche Sprecher sie sind. “ Und konkret wird spezifiziert: „In den gleichen Zeitungen können bestimmte Seiten der freien Meinungsäußerung offenstehen. Nur muß dann völlig klar sein, daß die betreffenden Herausgeber sich nicht mit jedem Standpunkt identifizieren, der dort in der Diskussion steht. “ ${ }^{29}$ Es wäre reizvoll, an Hand dieses Textes die Behandlung der sog. „Kölner Erklärung “ der Theologieprofessoren in der Bistumspresse zu untersuchen! Jedenfalls ergibt sich aus diesen Texten, daß das Konzept „Dialogforum“ im Sinne eines freien, beliebigen Meinungsaustausches nicht zutreffend sein kann.

In seiner Untersuchung „Die Redakteure der Bistumspresse“ hat Eckhard Bieger 1978 von drei verschiedenen Modellen im Selbstverständnis der Redakteure gesprochen: Das Marketing-Modell, das Kanzel-Modell und das Forums-Modell. Er wies schon damals nach, daß das AKP-Konzept des Dialogforums bereits im Ansatz nicht eindeutig ist, wenn in den „Grundsätzen und Richtlinien für die Bistumspresse " einerseits etwa gesagt wird, das Bistumsblatt verstehe sich als Zeitung der Diözesankirche, die alle Gläubigen miteinander verbindet (Forumsprinzip), andererseits der Bischof die Richtlinien für die grundsätzliche Haltung festlegt (Kanzel-Prinzip) und schließlich der freie Dialog zwischen Redaktion und Leserschaft gefordert wird (MarketingPrinzip). ${ }^{30}$ 
Der Vorsitzende der Deutschen Bischofskonferenz, Bischof Karl Lehmann, hat auf der Mitgliederversammlung der AKP im Oktober 1988 in Mainz ebenfalls die Funktionen des Forums und des Dialoges differenziert dargelegt. Einerseits stellt er fest: „Wir sind vielleicht zu sehr geneigt, gerade im Blick auf pastorale Defizite in unserer Zeit Presse und Medien allzusehr zu instrumentalisieren und sie unmittelbar in den Dienst der Pastoral zur Behebung seelsorglicher Mangelerscheinungen einzusetzen oder gelegentlich auch da und dort als ein Instrument zur Durchsetzung schwer zu vermittelnder Inhalte. Doch obgleich die Katholische Presse über die Konsensbildung auch meinungsbildend und öffentlichkeitswirksam ist, steht sie letztlich im Dienst der Kommunikation in der Kirche." Diese Kommunikation bezeichnet Lehmann als umfassend, einschließlich aller Einzelvorgänge. Andererseits unterscheidet er eine Binnensicht und eine Außensicht der Katholischen Presse und sagt wörtlich: „Die Binnensicht hat zur Voraussetzung, daß man seinen eigenen Standort mutig und klar einnimmt und ungeachtet aller Dialogbereitschaft mit anderen Gruppen das eigene Profil nicht verwischt. Innerhalb der bunten pluralistischen Welt sind wir nur durch ein deutliches und klares Gesicht erkennbar. " Und als eine der wichtigen Anforderungen an die Katholische Presse nennt er den „Mut zur geistigen Orientierung und Führung aus der Entschiedenheit des Glaubens ".

Ein Bistumsblatt kann nach CeP Forum für Wortmeldungen aller Glieder der Kirche sein, ist aber nicht Ort des Dialogs über verschiedene Lehrmeinungen. Der Begriff "Dialogforum" ist daher mißverständlich. Nach CeP ist kirchliche Presse (und dazu können oder müssen Bistumszeitungen mit bischöflichen Herausgebern gezählt werden) Kommunikationsmedium für die Verkündigung der Botschaft der Kirche. Dies muß sich heute zwar in einer pluralen Gesellschaft vollziehen und diese auch reflektieren; doch vom Auftrag her sind einer kirchlichen Presse andere Ziele (und Grenzen) gesetzt als der säkularen.

Der Forumscharakter einer Zeitschrift ist noch aus einer anderen Sicht heraus problematisch, selbst dann, wenn er so verstanden wird, daß alle Gruppierungen zu Wort kommen müssen, aber die Meinung der Kirche eindeutig herausgestellt wird. Zeitungen und Zeitschriften leben nicht zuletzt vom Identifizierungscharakter. Dieser kann lokalbezogen sein oder themenbezogen (worauf die Vielzahl der neuen Spezialzeitschriften hinweist), er ist aber auch richtungsbezogen. Jeder Leser sucht in seiner Zeitschrift Bestätigung seiner Meinung. Die Kirche ist inzwischen so plural geworden (in der sozialen Struktur, in der religiösen Alltagspraxis und in der theologischen Auffassung), daß für die vielerlei Gruppierungen das Basiselement Bistum nicht mehr konsensfähig ist. Von daher ergibt sich die Frage, ob nicht von einem weiteren Prinzip Abschied genommen werden muß: der Vorstellung von katholischen Zeitschriften als Massenblättern. Im Gutachten "Katholische Presse" hat Michael Schmolke diese Vorstellung hinsichtlich der Bistumspresse vertreten. Angesichts des ständigen Auflagen- und Reichweitenverlustes erhebt sich die Frage, ob Bistumspresse gleichfalls nur noch als Spezialpresse Zukunftschancen hat. Sie kann für eine umgrenzte Zielgruppe durchaus ihre Funktion erfüllen, wie andere (evtl. auch neue?) Organe für andere Zielgruppen.

Bistumspresse als Gesinnungspresse, die einen Standort bezieht (wie es auch in einer Werbeaktion der AKP heißt), die ihre Zielgruppe nach der Realität umgrenzt und mit überdiözesaner Thematik über Bistumsgrenzen hinaus kooperiert, könnte durchaus viel stärker als bisher das Zeitgespräch im innerkirchlichen Bereich und in der Gesellschaft bestimmen. Im sozial-caritativen Bereich hat die Bistumspresse dies in den vergangenen Jahrzehnten eindeutig bewiesen. Ohne sie wäre die Thematik der Entwick- 
lungshilfe, der Ausländerintegrierung, des Lebensschutzes nicht so gesellschaftlich und innerkirchlich ins Bewußtsein gehoben worden, wie es der Fall ist. Aber viele andere Zeitthemen fehlen. Fragen des Kriegs- und Friedensdienstes und der Kernenergie werden nur spärlich behandelt. In anderen Bereichen fehlt Kirche und Kirchenpresse offensichtlich Glaubwürdigkeit oder sie sind mit Vorurteilen belastet: Den Feldzug gegen die Pornografie, den Alice Schwarzer in ihrer Frauenzeitschrift geführt hat, hat Kirchenpresse nie begonnen. Fasten und Heilfasten wird den Frauenillustrierten abgenommen, während das Thema in der Kirchenpresse kaum beachtet wird.

Auch zum innerkirchlichen Bereich lassen sich aktuelle Zeitthemen auflisten, die in der Bistumspresse nicht vorkommen; etwa eine Untersuchung der freiformulierten Fürbitten, die in den Gottesdiensten oft mit unverantwortlichen politischen Akzenten formuliert werden, oder überhaupt die freie, oft allzu freie Gestaltung der Gottesdienste; oder die Art und Weise, wie in den einzelnen Gemeinden ganz verschieden auf die Erstkommunion und die Firmung vorbereitet wird; oder überholte Traditionen in den Verbänden (muß oder kann ein Pfarrer Schützenkönig werden?); oder Fragen der Verwendung der Kirchensteuermittel im Bistum (ein Bereich, der keineswegs eine kirchliche Lehre berührt). Es sind nicht nur oder nicht einmal vorrangig die Fragen der Empfängnisverhütung, der Betreuung der wiederverheirateten Geschiedenen, des Zölibats, die behandelt werden müßten. Eine solche inhaltliche Profilierung setzt jedoch eine andere redaktionelle Ausstattung voraus, als sie immer noch gegeben ist. Wenn vier Redakteure Woche für Woche 28 oder 32 Seiten füllen müssen, sind sie arbeitsmäßig so überlastet, daß sie die übergreifenden Themen nicht entsprechend recherchieren und darstellen können. Auch KNA kann dies nicht leisten, höchstens verstärkte Redaktionen in überdiözesaner Kooperation. Solche Kooperationen mit einem gemeinsamen überdiözesanen und einem bistumsbezogenen Teil können nicht am grünen Tisch geplant werden. Sie müssen aus den örtlichen Gegebenheiten erwachsen, aber in Angriff genommen werden, bevor einzelne Blätter wirtschaftlich nicht mehr gehalten werden können. Dabei genügt kein bloßes Zusammenlegen und Aufteilen der redaktionellen Aufgaben, sondern der gemeinsame überdiözesane Teil müßte von einer personell besser ausgestatteten Redaktion gestaltet werden, der Bistumsteil gleichfalls qualitativ (und nicht nur umfangmäßig) verbessert werden. Um neue Leser zu gewinnen, braucht Kirchenpresse ein neues Profil. Sie muß lebensnäher und nutzbarer sein. Dazu könnte auch eine Fernsehprogrammbeilage mit christlicher Orientierung beitragen.

Das Fazit dieser Überlegungen lautet: Ohne konzeptionelle Neuorientierung wird die Bistumspresse langsam, aber sicher dahinsiechen. Für Innovationen und Veränderungsmaßnahmen kann nicht die Frage ausschlaggebend sein, ob Verlage sie selbst finanzieren können, ob die bisherigen Verträge und Gegebenheiten es zulassen, ob die Redakteure und Verlagsleiter dies können, sondern ob die (bischöflichen) Herausgeber, ob die Kirche es wünscht und will. Wenn sie es will, wird es auch Wege geben, für die zwar Mittel bereitgestellt werden müssen und die auch das Risiko des Scheiterns einschließen; aber ohne neue Zielvorstellungen und ohne Risiko wird Hans Wagner mit dem Titel seiner dreibändigen Untersuchung von 1974 Recht behalten: „Das Ende der Katholischen Presse“. ${ }^{32}$ 


\section{Anmerkungen:}

1 In „Gemeinsame Synode oder Bistümer in der Bundesrepublik Deutschland“, Ergänzungsband Arbeitspapiere der Sachkommissionen, Freiburg 1977

2 Funktionsanalyse Bistumspresse, Media Markt Analysen, Frankfurt 1987. Die Auflagenentwicklungen gehen aus den vierteljährlichen Statistiken der AKP hervor.

3 Für die Gesamtentwicklung siehe: Michael Schmolke: Die schlechte Presse, Münster 1971; Ferdinand Oertel,Dialogforum Kirchenpresse, Limburg 1972

4 n Grundsätze für einen Rahmenplan der Kirchenpresse, Manuskriptdruck der Arbeitsgemeinschaft Katholische Presse, Bonn 1972

5 In "Gemeinsame Synode“ a.a.O.

6 Zusammenfassender Bericht über die Stellungnahmen 1-11 des Gutachtens Katholische Publizistik 72/73, Salzburg 1974

7 a.a.O.

8 a.a.O.

9 a.a.O.

10 a.a.O.

11 a.a.O.

12 a.a.O.

13 a.a.O

14 In "Gemeinsame Synode“, a.a.O.

15 In "Gemeinsame Synode", a.a.O.

16 Feldbefragung - Kommunikations- und Informationserwartungen der Katholischen Bevölkerung gegenüber kirchlichen Massenmedien - insbesondere der Kirchenpresse - Institut für Kommunikationsforschung, Wuppertal 1975

17 a.a.O.

18 Ferdinand Oertel: Informationsverhalten und -erwartungen der Katholiken, in: HerderKorrespondenz 2/1976, Freiburg

19 Projekte Modell-Entwicklung Kirchenzeitung, Manuskriptdruck Arbeitsgemeinschaft Katholische Presse, Bonn 1977

20 a.a.O.

21 a.a.O.

22 Wolfgang Seibel: Zwischenbilanz in Sonderdruck des Instituts zur Förderung des Publizistischen Nachwuchses, München 1988

23 Eckhard Bieger: Die Redakteure der Bistumspresse, München, Paderborn, Wien 1978

24 Manuskript Rundfunksendung Bayerischer Rundfunk, Kirchenfunk 1977

25 Funktionsanalyse, a.a.O.

26 Katholischer Literaturkalender, Freiburg 1926

27 Evangelische Kommentare 5/89

28 Pastoralinstruktion „Communio et progressio“ über die Instrumente der sozialen Kommunikation, Trier 1971

29 a.a.O.

30 a.a.O.

31 Manuskriptdruck AKP, Bonn 1988

32 Hans Wagner: Das Ende der Katholischen Presse, Aschaffenburg 1974 


\section{Summary}

Since 1963 the Catholic press in West Germany has continued to decline. Whereas at the end of 1963, the time of the Second Vatical Council, there were 22 diocesan papers with a total circulation of 2,4 million, by 1989 this had shrunk to 1,5 million. In order to stabilize the position of the diocesan papers. the Conference of Bishops set up the "Kontaktstelle Verlagskooperation" (Centre for Publishing Cooperation) in 1974, which gave rise later to the "MedienDienstleistungs-Gesellschaft“ (MDG) (Media Services co.). In 1977 the AKP and MDG presented a "Plan for the Development of a Church Paper Model“. At present the MDG, together with representatives of the diocesan press and in collaboration with the media centre of the German Conference of Bishops, is working on a TV supplement which, in programme reviews and accompanying articles, aims at providing a qualified guidance based on Christian values. In general, editing and publishing cooperation between individual diocesan papers took place in three stages of development. The diocesan papers of Limburg and Fulda began cooperating in 1971 and this spread to Mainz in 1976. Later the diocesan papers of Hildesheim and Berlin decided to work together and they were joined by Osnabrück and Paderborn. In 1986 these four diocesan papers started a monthly supplement with the title "Familienjournal“ (Family Journal). Since 1988 the "Familienjournal“ is being distributed with the diocesan papers in Aachen and in Rottenburg-Stuttgart. With regard to the principles laid down for the diocesan press - organ of local church and forum for dialogue - new considerations would seem to be in place. No doubt diocesan papers are a support for the local church but they fail in this function according as the number of readers drops and mainly the old are left. Hence it is becoming more meaningful to exploit the possiblities of transdiocesan cooperation, where each diocese plays its part. Apart from this, changes should be considered with regard to the principle of dialogue forum as formulated by the AKP. Although the basic ideas of the AKP relating to the forum character were taken from the pastoral instruction "Communio et progressio ", the context of the basis for forum and dialogue in the Catholic Church press was not given much attention. Basically the diocesan press must become closer to life and more useful: if a new way of thinking and new directions are not worked out in this regard, it will gradually lose all relevance.

\section{Résumé}

Depuis 1963 la presse catholique de la RFA évolue dans un sens négatif: Tandis que 22 journaux d'évéché avec un tirage total de 24000 exemplaires ont paru fin 1963 - au temps du Ilème Concile Vaticain -, le tirage a baissé à 150000 en 1989. Pour stabiliser la situation de la presse diocésaine, la Conférence des Evéques a fondé la „Kontaktstelle Verlagskooperation“, dont est issue la „Medien-Dienstleistungs-Kooperation“ (MDG) (Société Services Média). En 1977 AKP et MDG ont présenté le projet du „Développement d'un modèle de journal de l'Eglise“. En ce moment la MDG, en collaboration avec des représentants de la presse diocésaine et en coordination avec le Centre Média de la Conférence des Evéques d'Allemagne, développe un supplément de télévision qui, dans l'apercu des émissions à venir et dans des articles complémentaires, doit offrir une orientation qualifiée basée sur une éthique chrétienne. En général, la coopération rédactionelle et éditoriale entre les divers journaux diocésains s'est faite en trois étapes: Les journaux d'èveché de Limburg et Fulda ont commencé une coopération en 1971, qui a été étendue sur Mainz en 1976. Plus tard les rédactions des journaux d'évéché de Hildesheim et Berlin se sont décidées pour une coopération à laquelle se sont aussi rattachés Osnabrück et Paderborn. En 1986 ces quatre périodiques d'évéché ont développé un supplément mensuel au titre de „Familienjournal“ (Journal de la famille). Depuis 1988 le "Familienjournal“ est aussi inclus dans les journaux d'évéché d'Aachen et de Rottenburg-Stuttgart. Quant aux principes formulés pour la presse d'évéché, organe de l'Eglise locale et forume de dialogue, de nouvelles reflexions semblent souhaitables: Sans aucun doute, les journaux diocésains servent au renforcement de l'Eglise locale, mais parallèlement è un tirage baissant et un vieillissement des lecteurs, ils perdent cette fonction. Aussi la possibilité de coopérations interrègionales avec des parts pour chaque diocèse gange-t-elle en importance. En outre, une modification concernant le principe d'un forum de dialogue - comme c'ètait formulé par l'AKP - mérite réflexion. Bien que les idées fondamentales du concept AKP pour la 
forme de forum aient été empruntées à l'instruction pastorale "Communio et Progressio", on a moins tenu compte, cependant, de la base et de sa place dans le forum et le dialogue au sein de la presse catholique. Par principe, il faut que la presse diocésaine devienne plus actuelle et plus pratique - sans une orientation nouvelle avec une conception adéquate elle ira en dépérissant.

\section{Resumen}

Desde 1963, la prensa católica de la RFA ha sufrido un desarrollo negativo: Aparecido en 1963 en el tiempo del II Concilio Vaticano - 22 periodicos del obispado con un tiraje total de 2,4 millones de ejemplares, disminuyó en 1988 1,5 millones. Para estabilizar la situación de la prensa del obispado, la Conferencia de Obispos estableció en 1974 la "Kontaktstelle Verlagskooperation“ (Centro de Contacto de Coopercion entre Editores) de la que se ha desarrollado la Asociación para Servicios de Medios (ASM). En 1977, la AKP y la ASM presentaron el „Proyecto para desarrollo modelo de periódicos eclesiásticos“. Actualmente, la ASM en colaboración con represantantes de la prensa del obispado y de acuerdo con el centro de medios de la Conferencia de Obispos A lemana desarrolla un suplemento de televisión que pretende ofrecer una orientación cualificada para valores cristianos en el resumen de los programas y en articulos adjuntos. Generalmente, la cooperación de radactores y editoras entre determinados periódicos del obispado se realiza en tres niveles de desarrollo. Los periódicos de obispado de Limburg y Fulda, comenzaron en 1971 por una cooperación; esta cooperación, en 1976 fue extendida a Mainz. Más tarde los periódicos del obispado de Hildesheim y Berlin se decidieron por una cooperación, a la que se juntaron también Osnabrück y Paderborn. En 1986, estos cuatro periódicos de obispado desarrollaron un suplemento mensual bajo el titulo „Diario Familiar“. Desde 1988 este „Diario Familiar" también se anexa a los periódicos de obispado de Aachen y Rottenburg-Stuttgart. Vienen a ser necesarias nuevas reflexiones en cuanto a los perincipios del portavoz de la iglesia local, formuladas para la prensa del obispado: Indudablemente, los periódicos del obispado apoyan la consolidación de la iglesia local, sin embargo pierden esta función, si el tiraje está en descenso y la edad de los lectores sube. Por eso, la posibilidad de cooperación extradiocesana con participación diocesana va a ser cada vez más importante. Además, alternativas considerando el principio del forum de diálogos - como fue expresado por la AKP - merecen ser pensados. Por una parte, los pensamientos fundamentales del concepto de la AKP en torno al caracter de foro se tomaron de la instrución pastoral „Communio et Progressio“; pero por otra parte casi no se considera el contexto de la base, ni para el foro, ni para el diálogo en la prensa católica. Principalmente, la prensa del obispado debe inscribirse más cerca de la vida y de la utilidad - sin la adecuada reorientación conceptual va consumirse poco a poco. 